\title{
Efficacy of splinting and oral steroids in the treatment of carpal tunnel syndrome: A prospective randomized clinical and electrophysiological study
}

\author{
Sanjay Mishra, Sudesh Prabhakar, Vivek Lal, Manish Modi, Chandi P. Das, Dheeraj Khurana \\ Department of Neurology, PGIMER, Chandigarh, India
}

Objective: To study the efficacy of splinting and oral steroids in the management of carpal tunnel syndrome (CTS). Design: Prospective, randomized, open-label, clinical and electrophysiological study with 3-month follow-up. Materials and Methods: Forty patients with CTS were randomly divided into splint group (N-20), wearing splint in neutral position for 4 weeks; and steroid group (N-20), who received oral prednisolone $20 \mathrm{mg} /$ day for 2 weeks followed by $10 \mathrm{mg} /$ day for 2 weeks. Clinical and electrophysiological evaluations were done at baseline and at 1-month and 3month follow-up. Independent ' $t$ ' test and paired 't' test were used for statistical analysis. Outcome Measures: Primary outcome measure was the symptom severity score and functional status score. Secondary outcome measures were median nerve sensory and motor distal latency and conduction velocity. Results: At the end of 3 months, statistically significant improvement was seen in symptom severity score and functional status score in both groups $(P<0.001)$. Median nerve sensory distal latency and conduction velocity also improved significantly in both the groups at 3 months. Improvement in motor distal latency was significant $(P=0.001)$ at 3 months in steroid group, while insignificant improvement $(P=0.139)$ was observed in splint group. On comparing the clinical and electrophysiological improvement between the two groups, except for the functional status score, there was no significant difference at 3-month follow-up. Improvement in functional status score was significantly more in steroid group $(P=0.03)$. Conclusion: There was significant improvement in both groups, clinically as well as electrophysiologically, at 3 months. On comparing the efficacy of the two treatment methods, except for the functional status score, there was no significant difference between the two groups.

Key words: Carpal tunnel syndrome, clinical follow-up, electrophysiology, oral steroids, splinting

\section{Introduction}

Carpal tunnel syndrome (CTS) is the most common focal compressive neuropathy and is caused by entrapment of the median nerve in the carpal tunnel. Prevalence of electrophysiologically confirmed symptomatic CTS is about $3 \%$ among women and $2 \%$ among men, with peak prevalence in women older than 55 years of age. ${ }^{[1]}$ Early diagnosis and treatment of CTS are important because delay can result in irreversible median nerve damage with persistent symptoms and permanent disability. Treatment options include rest and avoidance of excessive activity at hand, exercises, laser therapy, splinting the wrist in neutral position, nonsteroidal anti-inflammatory drugs oral steroids, local corticosteroid injections and surgery to decompress the median nerve segment. ${ }^{[2]}$ Splinting the wrist in neutral position has been reported to provide symptomatic relief. ${ }^{[3,4]}$ Oral steroid therapy has also been found to result in significant decrease in symptoms of CTS in a few recent studies. ${ }^{[5-7]}$ However, data on comparative analysis of the conservative treatment modalities for CTS is limited in the literature. The present study aims at studying the effects of splinting in neutral position of the wrist and oral prednisolone on symptomatology and neurophysiological variables in patients with CTS.

\section{Materials and Methods}

A prospective randomized open-label clinical and electrophysiological study of efficacy of splinting and oral steroids for the treatment of CTS was done. Study population consisted of 66 patients who were diagnosed to have CTS in neurology outpatient department of a tertiary care center between July 2003 and December 2004. The study was approved by the ethical research committee of the Institute.

Patients with symptoms suggestive of carpal tunnel syndrome of at least 1-month duration and electrophysiological evidence of median neuropathy at wrist were included in the study. Patients 
were referred from internal medicine, plastic surgery and orthopedic outpatient departments of our institute and also by general practitioners from outside the hospital. The clinical criteria laid down by the American Academy of Neurology were used for diagnosis of CTS. ${ }^{[8]}$ The electrophysiological criteria used for the diagnosis of CTS included the presence of two or more of the following:

1. Median nerve motor distal latency recording at abductor pollicis brevis and stimulating at wrist greater than $4.4 \mathrm{~ms}$.

2. Median nerve antidromic sensory peak latency recording at digit II greater than $3.5 \mathrm{~ms}$.

3. Difference between antidromic median sensory latency and ulnar sensory latency at digit IV greater than $0.5 \mathrm{~ms}$.

4. Antidromic latency difference of $>0.5 \mathrm{~ms}$ between median nerve at digit II and ulnar nerve at digit V using the same distance of measurement.

The severity of symptoms and functional status was assessed using a scoring system as proposed by Levine et al. ${ }^{[9]}$ Symptom severity score has 11 items concerning pain, nocturnal symptoms, numbness, tingling and weakness. Functional status score has 8 items (difficulty in writing, buttoning, opening jars, holding a book, gripping telephone handle, household chores, carrying grocery bags, bathing and dressing). Each item of these scales has five responses ranging from 1 (no symptom or no difficulty) to 5 (very severe symptoms preventing the activity). Mean of the scores for all symptoms and activities was calculated and compared with scores at follow-up at 1 month and 3 months. All patients were randomly allocated to one of the following two groups:

1. Splinting in neutral position.

2. Oral steroid.

Randomization was done using the table of random numbers. Patients in splint group were advised to wear commercially available carpal tunnel splint at night and as much as possible during the daytime for 4 weeks. In the case of bilateral symptoms, both hands were treated. They were also told not use additional medicines or other methods of treatment during the study period.

Patients in steroid group were given oral prednisolone $20 \mathrm{mg} /$ day for 2 weeks followed by $10 \mathrm{mg}$ /day for another 2 weeks, as described in previous studies. ${ }^{6}$ Advice to avoid extremes of wrist flexion or extension, excessive hand movement and hand rest was common to both groups. The clinical outcome was assessed at 1 and 3 months. Primary outcome measures were the symptom severity score and functional status score. Secondary outcome measures were median nerve sensory and motor distal latency and conduction velocity. Normal values of the motor conduction velocity and sensory conduction velocity in our lab are $56 \pm 10$ $\mathrm{m} / \mathrm{s}$ and $55 \pm 12 \mathrm{~m} / \mathrm{s}$ respectively. Nerve conduction study was performed at baseline and also at 1- and 3-month follow-up.

\section{Exclusion criteria}

1. Patient with diabetes mellitus, trauma to wrist and deformity.

2. Any patient with evidence of generalized neuropathy/ radiculopathy on electrodiagnostic study.

3. Patients with advanced CTS having wasting, marked weakness with marked axonal loss on nerve conduction study or nonstimulatable nerves.

4. Patients with a history of peptic ulcer.

5. Patients treated previously for CTS using medical or surgical therapy.

6. Pregnant women with CTS.

7. Patients with systemic disorders like rheumatoid arthritis, hypothyroidism, amyloidosis, etc.

Nerve conduction study was performed on Medtronic machine using Keypoint software version 3.21. Motor and sensory conduction studies of median and ulnar nerve were performed on both hands. Motor conductions for median nerve were performed using the surface electrodes over abductor pollicis brevis (active) and first metacarpophalangeal joint (reference) while stimulation was done at wrist and elbow. For ulnar motor conductions, active electrode was placed at abductor digiti minimi and reference at the fifth metacarpophalangeal joint while stimulation was done at wrist, below elbow and above elbow. Antidromic sensory nerve conduction was performed using standard ring electrode recordings from digit II for median and digit $V$ for ulnar nerve. The recordings were made from ring finger with median and ulnar stimulation at wrist for digit IV sensory study. Parameters including distal latency, amplitude, conduction velocity and $\mathrm{F}$ responses were recorded.

All statistical calculations were performed using statistical package for social sciences (SPSS) for window version 10 . Unpaired 't' test was used to calculate statistical significance when comparing baseline parameters, improvement in clinical scores and change in electrophysiological parameters between the two groups. Chi-square test was used to compare sex distribution between the two groups at baseline. For comparison of variables within the groups between baseline and 1 month and 3 months post-treatment, paired ' $t$ ' test was used. $P$ value of less than 0.05 was considered significant.

\section{Results}

This study included 66 patients with 117 CTS-affected hands. Of the 66 patients, 26 did not fulfill the inclusion criteria and therefore were excluded. Of these, 13 patients had CTS secondary to conditions like diabetes mellitus, ${ }^{[2]}$ hypothyroidism, ${ }^{[6]}$ pregnancy, ${ }^{[3]}$ rheumatoid arthritis, ${ }^{[1]}$ amyloidosis. ${ }^{[1]}$ Remaining 13 patients had gross wasting, markedly decreased SNAP/CMAP amplitude or unrecordable SNAP/CMAPs in median nerve.

Remaining 40 patients (with 71 affected hands) were randomly allocated to two groups. Splint group had 20 patients with 36 affected hands and steroid group had 20 patients with 35 affected hands. Baseline characteristics of these patients are shown in Table 1. Mean age of the patients in the splint group was 42.91 years with a range of 23 to 60 years, while in steroid group it was 41.57 years with a range of 28 to 60 years [Table 1 ]. There was statistically no significant difference between the age and sex distribution of the patients in two groups. Overall, incidence of CTS was almost five times more in females compared to males. Electrophysiological abnormalities in both the groups at baseline 
Table 1: Baseline characteristics

\begin{tabular}{lccc}
\hline Parameter & $\begin{array}{c}\text { Splint group } \\
\text { (N-20 with 36 } \\
\text { hands) }\end{array}$ & $\begin{array}{c}\text { Steroid group } \\
\text { (N-20 with 35 } \\
\text { hands) }\end{array}$ & P-value \\
Age (years) & $42.91 \pm 9.39$ & $41.57 \pm 9.26$ & 0.54 \\
(mean \pm SD) & & $4 / 16$ & \\
Sex (male/female) & $3 / 17$ & $23.84 \pm 2.41$ & 0.67 \\
BMl (mean \pm SD) & $23.87 \pm 2.23$ & $6.31 \pm 7.50$ & 0.95 \\
Duration (months) & $6.40 \pm 7.09$ & & \\
(mean \pm SD) & & $1.68 \pm 0.31$ & 0.46 \\
FSS (mean \pm SD) & $1.74 \pm 0.34$ & $2.67 \pm 0.37$ & 0.10 \\
SSS (mean \pm SD) & $2.83 \pm 0.41$ & 40 & 0.56 \\
Tinel sign (\%) & 36.11 & 60 & 0.73 \\
Phalen sign (\%) & 63.88 & &
\end{tabular}

FSS- Functional status score, SSS- Symptom severity score. $P$-value $<0.05$ is significant.

are shown in Table 2. There were no statistically significant differences between the two groups.

Tables 3 and 4 show the outcome of treatment in both the treatment groups. In splint group, there was significant ( $P$-value $<0.001$ ) improvement in symptom severity score, functional status score and sensory distal latency at 1-month follow-up and the same was sustained at 3 -month follow-up. Sensory conduction velocity also showed statistically significant improvement at 3month follow-up.
In steroid group also, there was significant improvement in symptom severity score, functional status score at 1-month followup and the same was sustained at 3-month follow-up. Sensory and motor distal latencies and sensory conduction velocity showed statistically significant improvement at 3-month follow-up. Motor conduction velocity did not show significant improvement at 1- or 3-month follow-up.

Differences of the mean between baseline and 1 month and 3 month were compared between the two groups [Table 5]. Functional status score showed significant difference at 1 month and 3 month (better results in steroid group). Sensory conduction velocity also showed significant difference at 1 month, (more improvement in steroid group), but at 3 months the change from baseline was not significant between the two groups. Rest of the parameters including symptom severity score, motor distal latency, motor conduction velocity and sensory distal latency did not show any significant difference either at 1- or 3-month follow-up. Two patients in steroid group and one in splint group had worsening of symptoms at 3-month follow-up after initial response at 1 month.

Overall, significant improvement in primary outcome measures was observed at 1-and 3-month follow-up in both the groups and most of the electrophysiological data (secondary outcome

\section{Table 2: Electrophysiological abnormalities at baseline}

\begin{tabular}{lccc}
\hline $\begin{array}{l}\text { Electrophysiological } \\
\text { parameter }\end{array}$ & Splint group (36 hands) (mean $\mathbf{\pm}$ SD) & Steroid group (35 hands) (mean \pm SD) & $\boldsymbol{P}$-value \\
MDL (milliseconds) & $3.94 \pm 0.56$ & $3.94 \pm 0.98$ & 0.982 \\
CMAP amplitude (millivolts) & $8.36 \pm 2.34$ & $9.05 \pm 2.89$ & 0.274 \\
MCV (meters/second) & $54.13 \pm 14.65$ & $53.20 \pm 8.72$ & 0.749 \\
SDL (milliseconds) & $4.23 \pm 0.83$ & $3.88 \pm 1.02$ & 0.111 \\
SNAP amplitude (microvolts) & $22.86 \pm 9.70$ & $26.40 \pm 9.36$ & 0.123 \\
SCV (meters/second) & $33.04 \pm 8.69$ & $36.31 \pm 8.82$ & 0.121 \\
\hline
\end{tabular}

MDL: Motor distal latency, MCV: Motor conduction velocity, CMAP: Compound muscle action potential, SDL: Sensory distal latency, SCV: Sensory conduction velocity, SNAP: Sensory nerve action potential, $P$-value $<0.05$ is significant.

\begin{tabular}{lccccc}
\hline & \multicolumn{3}{c}{ Table $3:$ Treatment outcome: Splint group (N-36) } \\
\hline Parameters & Baseline & 1 month & 3 months & $\begin{array}{c}\text { Mean change (0-1) } \\
\text { mean } \pm \text { SD }(\boldsymbol{P} \text { value })\end{array} \quad \begin{array}{c}\text { Mean change (0-3) } \\
\text { mean } \pm \text { SD }(\boldsymbol{P} \text { value })\end{array}$ \\
SSS & $2.83 \pm 0.41$ & $2.48 \pm 0.52$ & $2.43 \pm 0.56$ & $0.34 \pm 0.42(<0.001)$ & $0.39 \pm 0.54(<0.001)$ \\
FSS & $1.74 \pm 0.34$ & $1.60 \pm 0.41$ & $1.57 \pm 0.43$ & $0.14 \pm 0.13(<0.001)$ & $0.16 \pm 0.17(<0.001)$ \\
MDL & $3.94 \pm 0.56$ & $3.88 \pm 0.66$ & $3.74 \pm 0.51$ & $0.06 \pm 0.77(0.607)$ & $0.20 \pm 0.79(0.139)$ \\
MCV & $54.13 \pm 14.65$ & $52.03 \pm 9.42$ & $50.73 \pm 7.46$ & $2.09 \pm 16.53(0.452)$ & $3.40 \pm 16.22(0.217)$ \\
SDL & $4.23 \pm 0.83$ & $4.07 \pm 0.86$ & $3.88 \pm 0.56$ & $0.16 \pm 0.63(0.136)$ & $0.35 \pm 0.76(0.009)$ \\
SCV & $33.04 \pm 8.69$ & $34.40 \pm 9.51$ & $35.87 \pm 9.06$ & $-1.41 \pm 7.11(0.24)$ & $-2.83 \pm 7.88(0.03)$ \\
\hline
\end{tabular}

SSS: Symptom severity score, SCV: Sensory conduction velocity, FSS: Functional status score, MCV: Motor conduction velocity, SDL: Sensory distal latency, MDL: Motor distal latency, 0-1: Difference of mean between baseline and 1 month follow-up, 0-3: Difference of mean between baseline and 3 months follow-up, $P$-value $<0.05$ is significant.

\begin{tabular}{lccccc}
\hline & \multicolumn{2}{c}{ Table 4: Treatment outcome: Steroid group (N-35) } \\
\hline Parameters & Baseline & 1 month & 3 months & $\begin{array}{c}\text { Mean change (0-1) } \\
\text { mean } \pm \text { SD }(\boldsymbol{P} \text { value })\end{array} \quad \begin{array}{c}\text { Mean change }(\mathbf{0}-3) \\
\text { mean } \pm \text { SD }(\boldsymbol{P} \text { value })\end{array}$ \\
SSS & $2.67 \pm 0.37$ & $2.27 \pm 0.48$ & $2.18 \pm 0.63$ & $0.40 \pm 0.30(<0.001)$ & $0.49 \pm 0.44(<0.001)$ \\
FSS & $1.68 \pm 0.31$ & $1.48 \pm 0.30$ & $1.45 \pm 0.35$ & $0.20 \pm 0.11(<0.001)$ & $0.23 \pm 0.17(<0.001)$ \\
MDL & $3.94 \pm 0.98$ & $3.74 \pm 0.73$ & $3.47 \pm 0.77$ & $0.19 \pm 0.76(0.143)$ & $0.47 \pm 0.73(0.001)$ \\
MCV & $53.20 \pm 8.72$ & $52.17 \pm 6.19$ & $54.01 \pm 5.64$ & $1.03 \pm 11.37(0.594)$ & $-0.80 \pm 8.72(0.588)$ \\
SDL & $3.88 \pm 1.02$ & $3.74 \pm 0.80$ & $3.32 \pm 0.64$ & $0.13 \pm 0.71(0.27)$ & $0.55 \pm 0.66(<0.001)$ \\
SCV & $36.31 \pm 8.82$ & $33.75 \pm 5.89$ & $39.82 \pm 6.47$ & $2.56 \pm 9.38(0.11)$ & $-3.51 \pm 8.48(0.02)$ \\
\hline
\end{tabular}

SSS - Symptom severity score, SCV- Sensory conduction velocity, FSS - Functional status score, MCV-Motor conduction velocity, SDL- Sensory distal latency, MDL- Motor distal latency, 0-1: Difference of mean between baseline and 1 month follow-up, 0-3: Difference of mean between baseline and 3 months follow-up, $P$-value $<0.05$ is significant. 
Table 5: Comparison of the outcome of the two groups

\begin{tabular}{lccc}
\hline Parameter & $\begin{array}{c}\text { Splint } \\
\text { (mean } \pm \text { SD) } \\
(\mathbf{N}-36)\end{array}$ & $\begin{array}{c}\text { Steroid } \\
\text { (mean } \pm \text { SD) } \\
(\mathbf{N}-35)\end{array}$ & $P$ value \\
SSS 0-1 & $0.34 \pm 0.42$ & $0.40 \pm 0.30$ & 0.52 \\
SSS 0-3 & $0.39 \pm 0.54$ & $0.49 \pm 0.44$ & 0.42 \\
FSS 0-1 & $0.14 \pm 0.13$ & $0.23 \pm 0.17$ & 0.01 \\
FSS 0-3 & $0.16 \pm 0.17$ & $0.26 \pm 0.21$ & 0.03 \\
MDL 0-1 & $0.06 \pm 0.77$ & $0.19 \pm 0.76$ & 0.46 \\
MDL 0-3 & $0.20 \pm 0.79$ & $0.43 \pm 0.70$ & 0.13 \\
MCV 0-1 & $2.09 \pm 16.53$ & $1.03 \pm 11.37$ & 0.75 \\
MCV 0-3 & $3.40 \pm 16.22$ & $-0.80 \pm 8.72$ & 0.18 \\
SDL 0-1 & $0.16 \pm 0.63$ & $0.13 \pm 0.71$ & 0.86 \\
SDL 0-3 & $0.35 \pm 0.76$ & $0.55 \pm 0.66$ & 0.25 \\
SCV 0-1 & $-1.41 \pm 7.11$ & $2.56 \pm 9.38$ & 0.047 \\
SCV 0-3 & $-2.83 \pm 7.88$ & $-3.51 \pm 8.48$ & 0.72
\end{tabular}

SSS: Symptom severity score, SCV: Sensory conduction velocity, FSS: Functional status score, MCV: Motor conduction velocity, SDL: Sensory distal latency, MDL: Motor distal latency, 0-1: Difference of mean between baseline and 1 month follow up, 0-3: Difference of mean between baseline and 3 month follow up, $P$-value $<0.05$ is significant.

measures) at 3-month follow-up, but except for functional status score, there was no significant difference in improvement when both the groups were compared.

None of the patients in steroid group reported any adverse effects, while in splint group, two patients reported discomfort and swelling of hand and wrist. Discomfort was temporary and it improved with persistent and proper use of the splint. Compliance to medication was excellent in steroid group, while three patients were not using splint regularly as prescribed. They were using the splint only 5 to 6 days per week instead of most of the time daily as advised. These three patients in splint group were also taking nonsteroidal anti-inflammatory drugs like nimesulide and diclofenac from the chemists or themselves because of persistent symptoms. None of the patients in steroid group was taking any other medication.

\section{Discussion}

This was a prospective study of the efficacy of two treatment modalities - splint and oral steroids - in patients with CTS. We chose oral steroids since steroid injections are known to cause mechanical or chemical nerve injury and several patients do not accept injections in the wrist. Age and sex distribution in this study is similar to what has been observed by studies from India and other countries. ${ }^{[5,10-12]}$ Overall positivity of tinel sign and phalen sign was 38 and $62 \%$ respectively. Positivity of tinel sign has been reported to vary from 9 to $73 \%$ and that of phalen sign varies from 10 to $73 \% .^{[13]}$ Murthy et al reported a positivity of $24.6 \%$ for tinel sign and $17.5 \%$ for phalen sign. ${ }^{[10]}$ The most common abnormality on electrodiagnostic study was sensory distal latency prolongation. Of 71 CTS-affected hands, $5.63 \%$ had normal absolute values of latencies, amplitude and conduction velocities, but the difference between median and ulnar sensory distal latencies was consistent with the diagnosis of CTS. ${ }^{[14]}$ Electrodiagnosis of CTS is likely to be missed in 10 to $25 \%$ of patients if such comparison studies are not performed. ${ }^{[15}$
In the splint group, there was significant improvement in the symptom severity scores and functional status scores at 1 month and the same was sustained at 3-month follow-up. Along with symptomatic improvement, there was significant improvement in sensory distal latency and sensory conduction velocity during the 3-month follow-up. However, there was no significant change in motor nerve conduction parameters, possibly because the motor conduction abnormalities itself were minimal at baseline. In the published literature, there are variable reports of success with splint use (31 to 67\%) in different studies. ${ }^{[4,16]}$ Gerristen et al reported a success rate of $31 \%$ (26 of 83 patients) with splinting. ${ }^{[16]}$

Patients in steroid group also showed significant improvement in clinical scores at 1- and 3-month follow-up. Sensory and motor distal latencies and sensory conduction velocities also showed significant improvement at 3-month follow-up. In a study by Herskovitz et $a l,{ }^{[7]}$ all the treated patients reported improvement at 2-week follow-up of treatment with oral steroids. However, this effect gradually waned off over 8 weeks in all of them. Chang $e t$ $a l^{[6]}$ compared a regimen of $20 \mathrm{mg}$ prednisolone per day for 2 weeks followed by $10 \mathrm{mg}$ daily for next 2 weeks, with $20 \mathrm{mg}$ per day of prednisolone for two weeks only. Improvement in Global Symptom Score in the 4-week treatment group was achieved in $66 \%$ of patients. In the 2 -week treatment group, the improvement was seen in $49 \%$ of patients. The difference was statistically not significant. Persistence of improvement was $74.20 \%$ in the 4 week group and $74.10 \%$ in the 2 -week group with no significant difference. At 1-year follow-up, improvement decreased to $51 \%$, suggesting relapse of symptoms in $17 \%$ of patients who had responded at 1 month.

On comparing the mean of the change from baseline to 1 and 3 month (0-1 and 0-3) between the two groups, there was no significant difference in symptom severity score, motor and sensory distal latencies and conduction velocities either at 1-or 3-month follow-up. However, functional status score showed significantly more improvement in steroid group at 3-month follow-up. To the best of our knowledge, there are no comparative studies of these two treatment methods in the published literature for comparison with our data. Based on these observations, we do not intend to propose that oral steroid therapy is the treatment of choice for CTS or that splinting is inferior to steroids. However, findings do suggest the need for further studies on this subject. While deciding about the best approach for treatment, one would also have to consider the adverse effects like Cushingoid features, avascular necrosis, gastrointestinal side effects, worsening of diabetes and hypertension, etc., which might develop due to repeated use of systemic steroids since the symptoms of CTS may be recurrent.

Two patients in the steroid group and one in the splint group had worsening of symptoms at 3-month follow-up after initial response at 1 month. In the study by Burke et $a l,{ }^{[4]} 17 \%$ of the patients reported worsening of symptoms between the 2 -week and 2-month follow-up after splint use. Chang et $a l^{[6]}$ also reported that at 1-year follow-up, $17 \%$ of patients who had responded at 1 month had a relapse of symptoms at 1-year 
follow-up. In fact, all the conservative methods of treatment for CTS do not have a sustained effect. In a recent study, splinting was combined with steroid injections and patients were followed up for 1 year. The study concluded that steroid injections and splinting are effective for relief of CTS symptoms but have a long-term effect in only $10 \%$ of patients. ${ }^{[17]}$ In the study by Gelberman et al, $89 \%$ of the patients with severe CTS (having constant numbness, weakness, atrophy or sensory loss) had recurrence within a year, while only $60 \%$ patients with mild CTS (intermittent symptoms and normal sensory and motor examination) had recurrence after splinting and local injection, suggesting that relatively advanced CTS is more likely to recur after conservative treatments. ${ }^{[18}$

The obvious limitations of this study are short follow-up and small number of subjects in both the groups. However, our study has some methodological superiority also - like prospective, randomized design and the use of well established validated clinical scores for clinical assessment. We do not consider placebo effect as a possibility for the improvement in our study since both clinical and electrodiagnostic measurements improved at the 3-month follow-up. Nonetheless, to be very sure, randomized, double blind, placebo-controlled study with long-term follow-up is needed.

\section{Conclusions}

Both the treatment methods (splint and oral steroids) were found to be effective. There was significant improvement in both groups clinically during follow-up at 1 and 3 months as well as electrophysiologically at 3 months. On comparing the efficacy of these two treatment methods, there was no significant difference at 3-month follow-up except for the functional status score. Improvement in functional status score was significantly more in steroid group. Oral steroids are safe and well tolerated at low dose in the short term. Studies with larger sample size and longer follow-up are suggested to establish the duration of beneficial effect, superiority of one treatment method over the other and actual relapse rates of symptoms.

\section{Acknowledgment}

We wish to thank Dr. Ritesh Aggarwal for his kind help in statistical analysis.

\section{References}

1. Atroshi I, Gummesson C, Johnson R, Ornstein E, Ranstam J, Rosen I. Prevalence of carpal tunnel syndrome in a general population. JAMA 1999;282:153-8.

2. Burke DT. Conservative management of carpal tunnel syndrome. Phys Med Rehabil Clin N Am 1997;8:513-28.

3. Kruger V, Kraft G, Deitz J, Amies A, Polssar L. Carpal tunnel syndrome: Objective measures and splint use. Arch Phys Med Rehab 1991;72:517-20.

4. Burke D'T, Burke MM, Steward GW, Combre A, Sholten RJ, Bouter LM. Splinting for carpal tunnel syndrome: In search of the optional angle. Arch Phys Med Rehab $1994 ; 75: 1241-4$.

5. Chang MH, Chiang HT, Lee SS.J, Ger LP, Lo YK. Oral drug of choice in carpal tunnel syndrome. Neurology 1998;51:390-3.

6. Chang MH, Ger LP, Hsieh PF, Huang SY. A randomized clinical trial of oral steroids in the treatment of carpal tunnel syndrome. A long-term follow-up. J Neurol, Neurosurg Psychiatr 2002;73:710-4.

7. Herskovitz S, Berger AR, Lipton RB. Low dose, short term oral prednisolone in the treatment of carpal tunnel syndrome. Neurology 1995;45:1923-5.

8. Quality Standards Subcommittee of the American Academy of Neurology. Practice parameter for carpal tunnel syndrome (summary statement). Neurology $1993 ; 43: 2406-9$

9. Levine DW, Simmons BP, Koris MJ, Datroy LH, Hohl GG, Fossel AH, et al. A selfadministered questionnaire for the assessment of severity of symptoms and functional status in carpal tunnel syndrome. J Bone Joint Surg (A) 1993;75:1585-92.

10. Murthy JMK, Meena AK. Carpal tunnel syndrome- Is it really uncommon in India. Neurol India $1995 ; 43: 26-8$

11. Goyal V, Bhatia M, Padma MV, Jain S, Maheshwary MC. Electrophysiological evaluation of 140 hands with earpal tunnel syndrome. J Assoc Physic India $2001 ; 49: 1070-3$

12. Murthy JK, Meena AK. Carpal tunnel syndrome-electrodiagnostic aspects of fifty seven symptomatic hands. Neurol India 1999;47:272-5.

13. Hennessey WJ, Kuhlman KA. The anatomy, symptoms and signs of carpal tunnel syndrome. Phys Med Rehabil Clin N Am 1997;8:439-57.

14. Jablecki CK, Andary MT, Floeter MK. Miller RG, Quartly CA, Vennix M.J, et al. of the American Association of Electrodiagnostic Medicine, American Academy of Neurology and the American Academy of Physical Medicine and Rehabilitation. Neurology 2002;58:1589-92.

15. Preston DC. Distal median neuropathy. Neurol Clin 1999;47:407-24.

16. Gerristen AA, Korthals-de Bos IB, Laboyrie PM, de Vet HC. Splinting for carpal tunnel syndrome: Prognostic indicators of success. J Neurol Neurosurg Psychiatr $2003 ; 74: 1342-4$.

17. Graham RG, Hudson DA, Solomons M, Singer M. A prospective study to assess the outcome of steroid injections and wrist splinting for the treatment of carpal tunnel syndrome. Plast Reconstr Surg 2004;113:550-6.

18. Gelberman RH, Aronson D, Weisman MH. Carpal tunnel svndrome: Results of a prospective trial of steroid injection and splinting. J Bone Joint Surg (Am) $1980 ; 62: 1181-4$

Accepted on 08-05-2006 